\title{
Simplified Fluorescence Microscopy of Tubercle Bacilli
}

\author{
By E. MATTHAEI \\ University of Melbourne, Australia
}

SUMMARY: The fluorescence of tubercle bacilli stained with auramine and rhodamine does not require ultra-violet light. It can be caused by blue light up to $496 \mathrm{~m} \mu$. A copper sulphate ammonia liquid filter, suitably diluted, will transmit this waveband. A gelatin screen filter, absorbing all light below $510 \mathrm{~m} \mu$. is used in the eyepiece. Normal, high intensity projection flament lamps, combined with lamp condensers of large aperture, provide suitable light sources. The numerical aperture of the microscope condenser must be fully used by immersion in glycerol. The fluorescence is very bright with the usual biological, and certain binocular, microscopes. The simplicity of the equipment enables fluorescence microscopy of tubercle bacilli to be used at low cost in any laboratory.

The phenomenon of fluorescence as applied to stained bacilli may be described as follows. Certain fluorochroms (e.g. auramine) absorb light of a certain wavelength, and convert it into light energy of another wave-length. This effect is observed by illuminating the specimen with suitable light passed through a filter which transmits a wave-band capable of causing fluorescence, and by incorporating into the observing part of the microscope a filter which transmits the colour of the fluorescence, but absorbs the light which has caused it.

The use of auramine as an acid-fast stain for the examination of tubercle bacilli in fluorescent light under the microscope was first introduced by Hagemann in 1988. Later workers made several improvements in Hagemann's method, but these were mainly technical. In essence the principle has remained the same, and is to-day recognized as having great advantages over the usual Ziehl-Neelsen technique: the staining process is quick and the result very clear-cut because of the great contrast between bacilli and background, the appearance of the field being almost that of a dark-ground with the bacilli a bright golden-yellow colour. Moreover, because of the distinctive appearance of the bacilli, a much lower magnification than usual can be used: $\times 300-400$ instead of the usual $\times \mathbf{1 3 5 0}$. Thus an area from ten to twenty times that of the average oil-immersion field can be covered, and the time of searching the slide correspondingly decreased.

The instruments used in the early development of fluorescence microscopy were equipped with a quartz optical system and a carbon arc with ultra-violet filter to produce the necessary fluorescence. Later workers (see Ellinger, 1940) considered that the fluorescence could be obtained with light of $300-400 \mathrm{~m} \mu$. wave-length, and since most of this is transmitted by the usual optical glass, the use of special quartz lenses and condensers became unnecessary. The mercury arc, with a very strong emission band at $365 \mathrm{~m} \mu$., is now used as the most practicable light source, together with a liquid copper sulphate ammonia filter to absorb all the light of more than $436 \mathrm{~m} \mu$.

Richards, Kline \& Leach (1941) described a high-intensity low-voltage 
filament lamp with high ultra-violet emission, together with a blue ultraviolet transmitting glass filter, which again simplified the equipment necessary to produce fluorescence,

Earlier, Keller (1938) had achieved fluorescence of tubercle bacilli by means of a glass filter with a transmission maximum in the violet, using a $6 \mathrm{~V} .5 \mathrm{amp}$. filament lamp as source of light. This arrangement, though simple, lacked intensity. Strugger (1939) described it as 'sehr lichtschwach'.

The purpose of the present paper is to show that adequate fluorescence of auramine can be produced by light up to $495 \mathrm{~m} \mu$. The whole of the blue part of the spectrum is thus used. With such a wide wave-band, most high-intensity projection-type filament lamps (readily obtainable everywhere) give sufficient intensity to cause good fluorescence.

Some confusion seems to exist about the photometry of fluorescence microscopes. I therefore investigated the problem of how best to utilize light sources and lamp condensers in combination with the numerical aperture of the microscope and its condenser.

\section{EXPERIMENTAL}

The kind of light needed for fluorescence was determined by first using a mercuryvapour lamp with a Wratten filter No. 50 and a $4 \mathrm{~cm}$. layer of $2 \%$ aqueous copper sulphate. This filter is practically monochromatic and transmits the blue violet light of $485 / 6 \mathrm{~m} \mu$. and a small amount of the violet $408 \mathrm{~m} \mu$. The tubercle smears were stained (see below) with auramine and rhodamine. A Wratten gelatin filter G15, absorbing all light below $510 \mathrm{~m} \mu$., was used as screen-filter in the eyepiece. With a magnification of $\times 400$, well-defined tubercle bacilli were seen against an almost black background.

Since, therefore, the $435 / 6 \mathrm{~m} \mu$. mercury lines are capable of producing the required fluorescence, a copper sulphate ammonia liquid filter was substituted for the Wratten filter No. 50. This liquid filter is described by Bowen (1942) in combination with a sodium nitrite filter for the isolation of the $435 / 6 \mathrm{~m} \mu$. lines. If used without the sodium nitrite filter and altered in concentration, it can be made to transmit the whole of the blue spectrum including the $492 / 6 \mathrm{~m} \mu$. lines. Tests in the Beckman spectrophotometer showed that the transmission goes down as far as $365 \mathrm{~m} \mu$.

The best working concentration of the filter was arrived at by diluting the copper sulphate ammonia solution until the $492 / 6 \mathrm{~m} \mu$. lines were just visible when seen through a hand spectroscope against a mercury-vapour arc. It is not claimed that the $492 / 6 \mathrm{~m} \mu$. lines themselves contribute to the fluorescence. But they certainly do not interfere with it, and make it possible to obtain greater intensities by using a transmission band up to $500 \mathrm{~m} \mu$. Since it is unnecessary to use a mercury lamp to produce light above $400 \mathrm{~m} \mu$, a normal microscope lamp, as used for critical illumination, was substituted. Its lamp was of the condensed-filament projection type (12 V., $100 \mathrm{~W}$.) in a suitable lamp-house with a 2 in. biconvex condenser. Results obtained with this lamp were very good.

The energy distribution of the projection filament lamps was ascertained. 
They had an average colour temperature of $2900^{\circ} \mathrm{K}$. (Pl. 1, fig. 1). Their radiant energy at $365 \mathrm{~m} \mu$. is only some $5 \%$, rising to $9 \%$ at $400 \mathrm{~m} \mu$. and to c. $28 \%$ at 500 . The actual energy contribution in the ultra-violet region below $400 \mathrm{~m} \mu$. is therefore very small. Even a $500 \mathrm{~W}$. projection filament lamp, used with an ultra-violet filter to isolate the $365 \mathrm{~m} \mu$. line, will not produce a noticeable fluorescence. It can be concluded that a normal projection filament lamp of sufficiently high intensity, used at its normal colour temperature and at its proper voltage, is capable of producing a good fluorescence with auraminestained tubercle bacilli, provided the transmission band of the filter is wide enough to let through enough blue light.

\section{The apparatus and its use}

The problem was investigated with a number of different microscopes, monocular and binocular, and with all readily available projection filament lamps used in a number of different lamp-housings. The following standard equipment is satisfactory:

Microscope. Monocular with a normal $4 \mathrm{~mm}$. objective, N.A. c. $0 \cdot 65$, primary magnification c. $\times 40$, eyepiece $\times 7$ or $\times 10$ with a G15 Kodak Wratten gelatin filter cut to size and placed over the field lens inside the eyepiece. (This position is better than that of the field stop, where irritating blemishes are clearly visible.) Condenser : normal Abbe two lens type, with N.A. 1.2. Filament lamp: $12 \mathrm{~V} ., 100 \mathrm{~W}$.

Lamp-housing (Pl. 1, fig. 2). Tubular with biconvex condenser lens $f=50 \mathrm{~mm}$., diameter $=50 \mathrm{~mm}$.

Filter $(A)$ (Pl. 1, fig. 3). The tube is made of phenolic resin, $7 \cdot 6 \mathrm{~cm}$. outside diameter, $6.3 \mathrm{~cm}$. inside diameter, $5 \mathrm{~cm}$. long; two rubber rings form a liquidtight joint with the glass plates $3 \mathrm{~mm}$. thick; two bakelite plates with appropriate apertures hold the filter together with four metal rods.

Filter (B) (Pl. 1, fig. 4). Alternative to $(A)$, above, an $8 \mathrm{oz}$. white glass, plane-walled medicine bottle, outside measurements $1 \frac{3}{4} \times 2 \frac{1}{2} \times 7$ in., inside clearance $c .1 \mathrm{in}$. Such a bottle is adequate as a filter, though not as good as $(A)$. The liquid in the filters has the following compositions: $\mathrm{CuSO}_{4} .5 \mathrm{H}_{2} \mathrm{O}, 8 \cdot 33 \mathrm{~g}$; conc. aqueous ammonia (sp.gr. $0 \cdot 880$ ), $100 \mathrm{ml}$.; water (for $5 \mathrm{~cm}$. layer thickness, filter $A$ ), $333 \mathrm{ml}$.; or (for $2 \cdot 5 \mathrm{~cm}$. layer thickness, filter $B$ ), $166 \mathrm{ml}$.

These filter concentrations are given as a guide. In individual cases they depend on such factors as the colour temperature and blue emission of the lamp. The best gauge of the filter concentration is the appearance of the stained smear. It should be such that golden-yellow tubercle bacilli are seen against a drab olive-green background. Dilution of the copper sulphate ammonia solution with water increases the width of the transmission band of the filter and the intensity of the field until the end of the blue part of the spectrum is reached. Further dilution quite suddenly changes the appearance of the background from a dark olive-green to a luminous blue-green, against which the fluorescent bacilli are difficult to distinguish.

Condenser. In normal microscopy the iris diaphragm of the condenser is used to bring about a balance between the desired definition and the required 
depth of focus. Therefore, except with oil-immersion objectives, the aperture of the condenser is never fully used. The system is entirely different in fluorescence microscopy. The fluorescing particles are self-luminous, and, independently of the condenser, they will fill the whole aperture of the objective. In consequence, no amount of 'stopping down' will increase the definition, nor influence the depth of focus. The condenser is in fact used to get as great an intensity of blue light as possible to the specimen. The average condenser has N.A. 1.2 if there is an immersion medium of a refractive index of not less than 1.2 between condenser and slide; when the condenser is used dry, the resulting N.A. cannot be more than 1. Since the light-gathering power of a condenser is proportional to the square of its N.A., an immersed condenser of N.A. 1.2 will transmit $44 \%$ more light than when dry, and a condenser with N.A. 1.4 will produce $96 \%$ more light. Cedarwood oil, because it has a fluorescence of its own, is not advisable as an immersion medium. Glycerol $\left(n_{D}=1 \cdot 463\right)$ is non-fluorescent and suitably viscous. The condenser should always be used with the iris diaphragm wide open. Canada balsam, still the usual cementing medium for optical surfaces, has, like cedarwood oil, a fluorescence of its own which increases with age. Complex condensers with many cemented surfaces are therefore to be avoided.

The same considerations apply to the photometry of the objectives as to that of the condensers. Taking a final magnification of $\times 400$ as a basis, a $4 \mathrm{~mm}$. objective $\times 40$, N.A. 0.65 , with an eyepiece $\times 10$ will yield $c .2 \frac{1}{2}$ times the amount of light as will an $8 \mathrm{~mm}$. objective $\times 20$, N.A. $0 \cdot 40$, with a $\times 20$ eyepiece. These results were confirmed by measurements with a photoelectric cell. With dry objectives, a cover-glass which may be loosely placed over the smear, is normally required. Alternatively, objectives specially corrected for use without cover-glass are needed for uncovered specimens.

The lamp. As an optical instrument the lamp must fulfil the following conditions. An image of the lamp filament is projected into the plane of the microscope condenser diaphragm. It must be of a size to fill the whole of the back lens of the condenser. The standard lamp mentioned earlier has a biconvex lens of $50 \mathrm{~mm}$. diameter and $50 \mathrm{~mm}$. focal length to produce this image. This corresponds to a numerical aperture of $0 \cdot 45$, and again conforms to the guiding rule to get as much light as possible into the microscope. A very efficient lamp can be constructed from a suitable tin container, with a spherical flask as a condenser (P1. 1, fig. 4). With a $250 \mathrm{~W}$. $115 \mathrm{~V}$. tubular projection filament lamp, enough light is obtained even for a binocular microscope. But to achieve this result it is essential that the projection filament lamps be used at their proper voltage. A diminution in voltage, though it will prolong the life of the filament lamp, will decrease the colour temperature considerably and the blue part of the spectrum will suffer most of this loss.

Other microscopes. Monocular microscopes, at the same magnifications and N.A.'s of objective and condenser will produce fluorescent images of practically the same intensity. There are small contributory factors, such as bloomed lenses which transmit more light, and the use of an aluminized mirror instead of a back-silvered one. The general result is not greatly influenced by these. 
Fluorite lenses, because of internal fluorescence, have not been found very satisfactory.

Binocular microscopes differ very considerably in their construction. The optical mechanism which splits the image into its two fields is made up of prisms. The number. of prisms and the number of air/glass surfaces affect the light transmission, which differs widely in binocular microscopes of various makes. This loss of light is of no great importance in normal microscopy, but it is disastrous for the fluorescence technique when used with the intensities and light sources outlined in this paper. With binocular microscopes, the best results were obtained with the Zeiss $\mathrm{L}$ type (Pl. 1, fig. 2). With aplanatic condenser N.A. 1.4, lamp-house with spherical flask as condenser (Pl. 1, fig. 4), and a projection filament lamp of $250 \mathrm{~W}$., $150 \mathrm{~V}$. a good binocular image is obtained. The Watson Service binocular microscope, under otherwise the same conditions, produces only a faint image. Other makes of instruments, as far as they were available for test, fell in between the above two extremes. Though a dark room is not necessary for this system of fluorescence microscopy, a cover over lamp, filter-bottle and stage is advisable for protection from extraneous light.

\section{Staining technique}

Since Hagemann's use of auramine in 1938, a number of variations of his staining technique have been recommended, all aiming at an increase in intensity of the fluorescence. Hughes (1946) described a combination of auramine, rhodamine and acridine yellow which gives a very good orange-red fluorescence. The commercially obtainable auramine and rhodamine are satisfactory; the acridine yellow will give indifferent results unless carefully prepared. The Department of Bacteriology of the Melbourne University has for several years used the following combination of auramine and rhodamine with success. The stain is prepared by mixing Auramine OS (Imperial Chemical Industries Ltd.) 3 g., Rhodamine BS (Imperial Chemical Industries Ltd.) $1.5 \mathrm{~g}$., and glycerol (Merck) $150 \mathrm{ml}$. Phenol crystals liquefied at $50^{\circ}(20 \mathrm{ml}$.) are added, and then $100 \mathrm{ml}$. distilled water. The stain, heated to $50-60^{\circ}$, is poured on to smears fixed at a temperature of $70-75^{\circ}$, left for $10 \mathrm{~min}$. and washed off with tap water. The preparation is decolorized with $0.5 \%(\mathrm{v} / \mathrm{v})$ conc. hydrochloric acid in $70 \%(v / v)$ ethanol in water, using three changes, the first for $1 \mathrm{~min}$., and the second and third for $2 \mathrm{~min}$. each. The slide is dried on a hot plate at $\mathbf{5 2}^{\circ}$.

Acknowledgement is due to the Department of Bacteriology of the Melbourne University for the preparation of the slides and description of the staining technique. I also wish to thank Assoc. Prof. W. A. Rawlinson for the evaluation of the blue filter transmission band.

\section{REFERENCES}

Bowen, E. J. (1942). The chemical aspects of light. Oxford: Clarenden Press.

El.uinger, P. (1940). Fluorescence microscopy in biology. Biol. Rev. 15, 323.

Hagemann, K. H. (1938). Fluoreszenzfärbung von Tuberkelbakterien mit Auramin. Münch. med. Wschr. 85, 1066.

HughEs, G. C. (1946). An improved stain for fluorescence microscopy. Tubercle, 91. 
Krels.er, Ch. J. (1938). Eine vereinfachte Methode zur Auffindung von Tuberkelbazillen im Fluoreszenzlicht. Münch. med. Wschr. 85, 2024.

Richards, O. W., Kuine, E. K. \& Leach, R. E. (1941). Demonstration of tubercle bacilli by fluorescence microscopy. Amer. Rev. Tuberc. 44, 255.

StrugGer, S. (1939). Die Anwendung der Luminiszenzmikroskopie in der Botanik. Zeiss Nachr. Jena, 1, 69.

\section{EXPLANATION OF PLATE}

Fig. 1. Energy distribution chart of high-intensity projection filament lamps showing proportion of ultra-violet and blue emission.

Fig. 2. Binocular microscope set up for fluorescence microscopy with filter vessel and microscope lamp normally used for critical illumination.

Fig. 8. Vessel for copper sulphate ammonia filter with phenolic resin spacer, glass covers and bakelite end-plates.

Fig. 4. Simple arrangement for observation of fluorescence with projection filament lamp in tin container, spherical flask as lamp condenser, and medicine bottle as filter vessel.

(Received 16 December 1949) 
Journal of General Microbiology, Vol. 4, No. 3

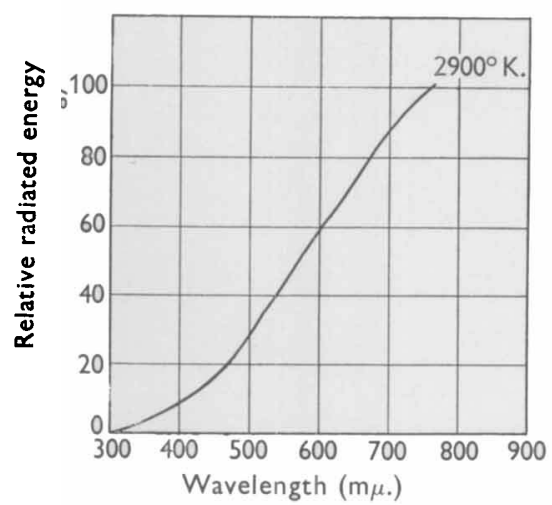

Fig. 1

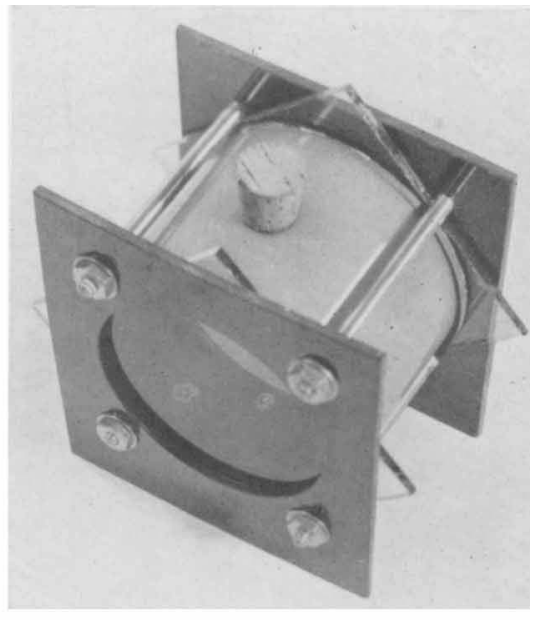

Fig. 3

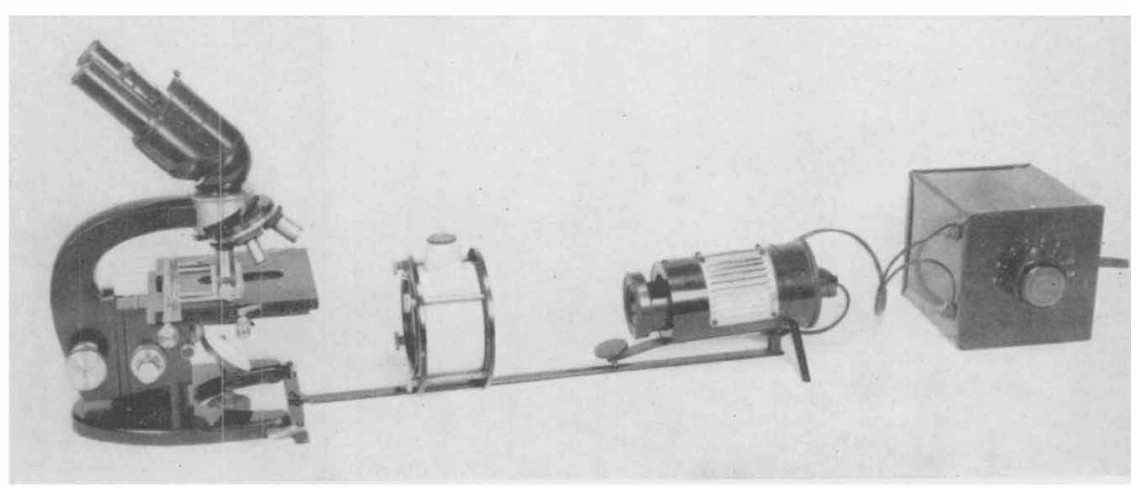

Fig. 2
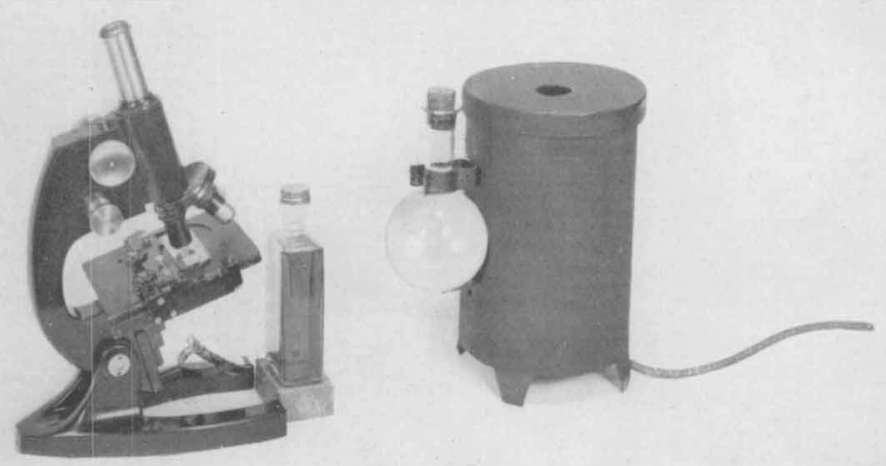

Fig. 4

E. MATthati-Simplified FluORESCENCE MiCRoscopy OF TUBERCle BaCILLI. Plate 1 